\title{
Systematic Review of Factors Affecting Quality of Life After Cytoreductive Surgery with Hyperthermic Intraperitoneal Chemotherapy
}

\author{
Maleen Leimkühler, MD ${ }^{1}$, Judith E. K. R. Hentzen, MD ${ }^{1}$, Patrick H. J. Hemmer, MD ${ }^{1}$, Lukas B. Been, MD, PhD ${ }^{1}$, \\ Robert J. van Ginkel, MD, $\mathrm{PhD}^{1}$, Schelto Kruijff, MD, $\mathbf{P h D}^{1}$, Barbara L. van Leeuwen, MD, $\mathbf{P h D}^{1}$, and \\ Geertruida H. de Bock, PhD $^{2}$ \\ ${ }^{1}$ Division of Surgical Oncology, Department of Surgery, University of Groningen, University Medical Center Groningen, \\ Groningen, The Netherlands; ${ }^{2}$ Department of Epidemiology, University of Groningen, University Medical Center \\ Groningen, Groningen, The Netherlands
}

\begin{abstract}
Background. Previous studies have shown that, overall, quality of life (QoL) decreases within the first 3-6 months after cytoreductive surgery with hyperthermic intraperitoneal chemotherapy (CRS + HIPEC), returning to baseline levels by 6-12 months. This systematic review aims to evaluate the factors affecting QoL after CRS + HIPEC within 12 months of surgery.

Methods. Electronic databases were investigated searching for articles reporting QoL with validated questionnaires up to September 2019. Risk of bias was assessed with the methodological index for non-randomized studies tool. The primary outcomes were short-term $(<6$ months after surgery) and medium-term (6-12 months after surgery) determinants of QoL after CRS + HIPEC. Secondary outcomes were QoL and reported symptoms over time.

Results. We included 14 studies that used 12 different questionnaires. The reported data were collected prospectively or retrospectively for 1556 patients (dropout $<50 \%$ in four studies). Overall, studies showed diminished QoL within 3 months after surgery and a recovery to baseline or
\end{abstract}

Electronic supplementary material The online version of this article (https://doi.org/10.1245/s10434-020-08379-9) contains supplementary material, which is available to authorized users.

(C) The Author(s) 2020

First Received: 23 December 2019;

Published Online: 26 April 2020

G. H. de Bock, PhD

e-mail: g.h.de.bock@umcg.nl greater by 12 months. QoL was negatively influenced by higher age, female sex, prolonged operation time, extensive disease, residual disease, adjuvant chemotherapy, complications, stoma placement, and recurrent disease. QoL results were comparable between studies, with dropout rates above and below 50\%.

Conclusions. QoL returns to baseline levels within 12 months after CRS + HIPEC provided the disease does not recur, and this recovery process is influenced by several factors.

Peritoneal metastases are present in advanced stages of several abdominal tumors. If left untreated, they are associated with poor prognosis, high morbidity, and reduced quality of life (QoL) ${ }^{1-4}$ Peritoneal metastasis was once considered incurable and suitable for only palliative treatment. $^{5-7}$ However, in carefully selected patients with limited and resectable disease and who have no distant metastases, research has shown that aggressive cytoreductive surgery (CRS) with hyperthermic intraperitoneal chemotherapy (HIPEC) may be considered a curative treatment option. ${ }^{8}$ In this extensive surgical procedure, all macroscopic disease is removed from the abdominal cavity, which is then perfused with heated chemotherapy agents. $^{9-11}$ Since the introduction of the CRS + HIPEC approach, multiple studies have shown improved prognosis in appropriately selected patients with peritoneal metastasis from various tumors. Today, CRS + HIPEC is even regarded as the standard of care for patients with peritoneal metastasis of colorectal origin or in those with pseudomyxoma peritonei..$^{5,12,13}$ 
Despite the undoubted success of CRS + HIPEC, the approach remains a high-risk treatment with a mortality of up to $8 \%$ and a morbidity of $23-66 \%$, even in experienced centers. ${ }^{9,14-19}$ Clinicians and patients must therefore seriously weigh the potential survival benefits against the substantial risk of treatment-related morbidity, mortality, and a potentially diminished QoL and functional status. Supporting this latter consideration, two systematic reviews were recently published in which it was suggested that patients who underwent CRS + HIPEC experienced a decrease in QoL, although this eventually returned to baseline levels within 12 months of surgery. ${ }^{20,21}$ However, both reviews relied on limited literature searches, and only one reported a range of QoL domains. ${ }^{21}$ Furthermore, neither review gave sufficient consideration to the determinants of QoL after CRS + HIPEC, such as stoma placement, ${ }^{22}$ disease recurrence, ${ }^{22}$ and dropout rates. Note that dropout rates can be highly selective and lead to the most ill patients in a cohort being underrepresented. In turn, this indicates the need for a further review of the factors affecting change in QoL after CRS + HIPEC in patients with peritoneal metastasis.

The objective of this systematic review is to identify the factors affecting QoL after CRS + HIPEC in patients with peritoneal metastasis over both the short-term (within 6 months of surgery) and the medium-term (6-12 months after surgery).

\section{MATERIALS AND METHODS}

We conducted a systematic review and reported the results according to the Preferred Reporting Items for Systematic Reviews and Meta-Analyses (PRISMA) and the Meta-analysis of Observational Studies in Epidemiology (MOOSE) guidelines. ${ }^{23,24}$

\section{Literature Search Strategy}

The search strategy was developed in collaboration with an experienced medical research librarian, and a full description of the strategy can be found in Supplementary Table 1. The systematic literature search was conducted in October 2018 and updated in September 2019, using the databases of PubMed, Embase, Cochrane library (trials), and Web of Science. We aimed to identify prospective and retrospective observational studies and randomized clinical trials (RCTs) that met predefined eligibility criteria. The Medical Subject Headings (MeSH) term "Quality of Life" and different terms for "HIPEC" were used. Only original peer-reviewed research was included. No further restrictions were placed on the study design, language, or study date. Finally, the references of included articles and related review articles were manually screened to identify additional relevant studies.

\section{Eligibility Criteria}

Studies were included for review based on the following eligibility criteria: (1) patients were treated with CRS + HIPEC, (2) reported QoL data were obtained by validated questionnaires, (3) patients with colorectal peritoneal metastasis were included, (4) data collection was prospective, and (5) research was original and peer-reviewed. To guarantee that the publications were understood at an academic level, the articles were required to be written in English, Dutch, German, or Spanish. We excluded any studies in which patients were retreated with HIPEC or another intraperitoneal chemotherapy (e.g., postoperative intraperitoneal chemotherapy). Studies reporting data only about patients with pseudomyxoma peritonei or a primary malignancy of the appendix or stomach were also excluded because of the different prognosis compared with other CRS + HIPEC indications. Finally, studies with a cross-sectional design were excluded because one measurement point is insufficient to measure the change in QoL.

\section{Study Selection}

Titles and abstracts were independently reviewed for eligibility according to predefined criteria by two authors (M.L. and J.H.). The reviewers were not blinded to publication date, journal, or authors. The full texts of potentially eligible articles were retrieved and assessed for inclusion independently by each author. Disagreement about study inclusion was resolved by consensus or by discussion with a third author (B.L.).

\section{Data Extraction and Quality Assessment}

Data extraction for predetermined items was performed independently by two authors (M.L. and J.H.). The following data were extracted: first author, publication year, country of origin, study years, study design, number of patients, inclusion and exclusion criteria, age, sex, tumor origin, morbidity and mortality related to CRS + HIPEC, QoL instruments used, measurement points, questionnaire response rate, and mean overall and subscale QoL scores (e.g., physical health, social health, emotional health, functional health, and cognitive health). If data were not reported, items were recorded as "NR" (not reported).

To evaluate the quality of the included articles, two reviewers (M.L. and J.H.) independently conducted a risk of bias analysis using the methodological index for nonrandomized studies (MINORS) for individual studies. ${ }^{25}$ In 
the event of disagreement, consensus was reached through discussion or by consulting a third author (B.L.). The MINORS criteria were specifically developed for use with studies that have a surgical intervention. Each item can be scored as 0 (not reported), 1 (reported but inadequate), or 2 (adequately reported), resulting in global ideal scores of 16 for noncomparative studies and 24 for comparative studies. Although the tool gives an indication of the quality of studies in different domains, it has no defined cut-off scores for what constitutes high or low quality.

The interobserver reliability of the risk of bias assessment was calculated using the intraclass correlation coefficient (ICC). The ICC for interobserver reliability was interpreted according to the definition of Landis and $\mathrm{Koch}^{26}$ as follows: poor if $<0.00$, slight if $0.00-0.20$, fair if $0.21-0.40$, moderate if $0.41-0.60$, substantial if $0.61-0.80$, and almost perfect if $0.81-1.00$.

\section{Outcomes}

The primary outcomes were the short- ( $<6$ months) and medium-term (6-12 months) factors affecting QoL in patients with peritoneal metastasis after CRS + HIPEC. The secondary outcome was the QoL after CRS + HIPEC in various domains after CRS + HIPEC, including overall health, physical health, emotional health, social health, functional health, and cognitive functioning, and symptoms.

\section{RESULTS}

\section{Study Selection}

A total of 1759 potentially relevant records were identified from four databases (Fig. 1). After removing duplicates, titles and abstracts of the remaining 869 records were screened. This resulted in 31 full-text articles being eligible for inclusion in our review. We then excluded 17 articles based on the eligibility criteria, leaving 14 articles that met all eligibility criteria for the systematic review.

\section{Study Characteristics}

QoL data were included for 1556 patients who had undergone CRS + HIPEC for peritoneal metastases due to a range of primary tumors (Table 1). ${ }^{22,27-39}$ There were 12 prospective and 2 retrospective studies; 8 were conducted in North America, 5 in Europe, and 1 in Asia. The studies used 12 different types of validated questionnaire to assess QoL after CRS + HIPEC. Most common among these were the Functional Assessment of Cancer Therapy (FACT), the European Organization for Research and
Treatment of Cancer (EORTC) QLQ-C30, the Medical Outcomes Study Health Survey Short Form (SF-36), and the Eastern Cooperative Oncology Group (ECOG) performance status (Supplementary Table 2). Most prospective studies conducted measurements at baseline and at 3, 6, and 12 months after surgery. Characteristics of the CRS + HIPEC procedures performed in the different studies can be found in Supplementary Table 3.

Excluding three studies, ${ }^{29,31,33}$ dropout rates were $9-51 \%$ at 6 months and $10-75 \%$ at 12 months (Fig. 2) $22,28,30-32,34-38$ and were mostly explained by the high mortality of $3-18 \% .^{22,28,30,34,36,37}$ Other reasons for dropout were mentioned in one study, including that patients were too sick $(13 \%)$ or refused $(6 \%)$ to participate further, but with no reason recorded in many cases $(32 \%) .^{30}$

\section{Quality and Risk of Bias Assessment}

To evaluate the quality of the included articles, we performed a risk of bias assessment using the MINORS criteria, the results of which are summarized in Supplementary Table $4 .^{25}$ All of the included studies were observational, and none reached the global ideal scores. The mean MINORS score was 9.83 (range 6-11) for the noncomparative studies and 14.50 (range 14-15) for the comparative studies. None of the included studies reached the maximum scores because their aims were unclear or because we could not determine whether prospective data collection was performed according to a previously reported protocol. However, 12 studies reported their endpoints in an adequate manner. Loss to follow-up was not reported in 3 studies $(16 \%)$, but it was reported in the other 11 studies (84\%), where it exceeded the 5\% limit applied by the MINORS criteria. Note that loss to followup $>5 \%$ is common in QoL research. We therefore evaluated the quality of the studies as mediocre. The ICC between the two reviewers, 0.95 , was almost perfect.

\section{Development of QoL During Follow-Up}

The general picture after CRS + HIPEC was for QoL to decrease over the first 3 months, to begin to recover by 6 months, and to reach or exceed the baseline measurements by 12 months (Fig. 3). ${ }^{22,27-31,34-38}$ This change in QoL was also evident in the different QoL domains, including physical health, ${ }^{22,27-38}$ emotional health, ${ }^{22,27-38}$ social health, ${ }^{22,27-34,36-38}$ functional health, ${ }^{22,27-35,37,38}$ and cognitive health (Fig. 4). ${ }^{22,27,29,37}$ Symptoms that most frequently arose or worsened within the first 6 months after surgery were fatigue, dyspnea, insomnia, and diarrhea. However, all symptoms except for diarrhea had improved by 12 months after surgery (Table 2). ${ }^{22,27,28,30,34,35,37}$ 


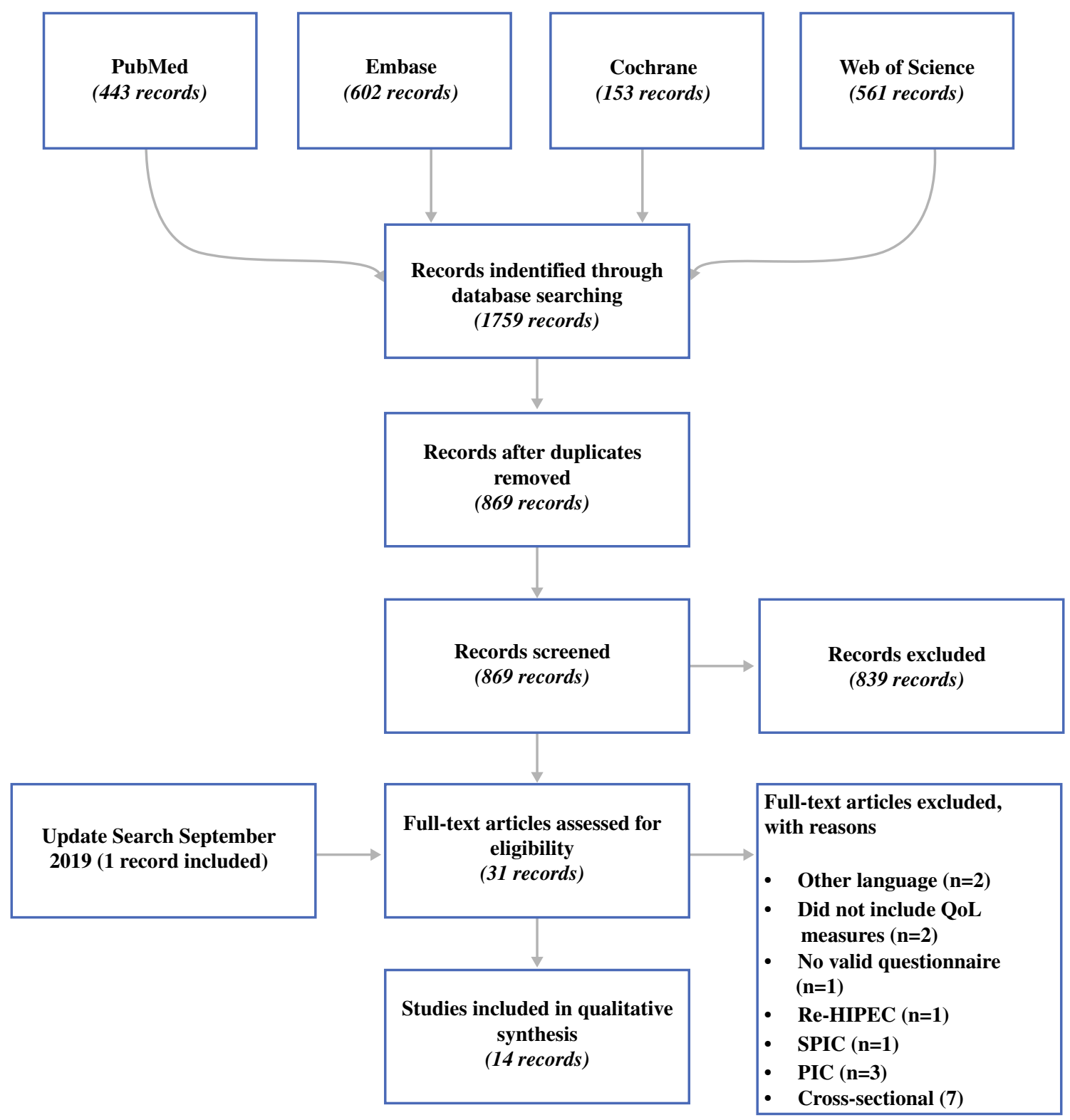

FIG. 1 Flowchart of systematic review. HIPEC hyperthermic intraperitoneal chemotherapy, IPC intraperitoneal chemotherapy, $Q o L$ quality of life, SPIC sequential perioperative intraperitoneal chemotherapy

Several studies described an increased level of pain 3 months after CRS + HIPEC compared with baseline levels. Thereafter pain levels decreased, reaching levels lower than baseline at 6 months. ${ }^{28,30,34,35,37}$

\section{Determinants of $Q o L$}

A variety of patient-, tumor-, and treatment-related factors were evaluated to determine their impact on $\mathrm{QoL}$ after CRS + HIPEC (Table 3). ${ }^{22,27,29,}$ 30,32,33, 35,36,38,39 Factors that negatively influenced QoL after CRS + HIPEC were higher age, ${ }^{32,33}$ female sex, ${ }^{27,32}$ prolonged operation time, ${ }^{22,36}$ high completeness of cytoreduction
(CC) score, ${ }^{22,32,33,36}$ treatment with adjuvant chemotherapy, ${ }^{22,33}$ postoperative complications, ${ }^{29,38}$ presence of a stoma, ${ }^{22,32,36}$ and disease recurrence within 12 months. ${ }^{22,36}$ A high Peritoneal Cancer Index (PCI) before CRS + HIPEC also influenced the QoL negatively, ${ }^{22,32,36}$ although one study found a statistically nonsignificant negative effect of the PCI on QoL in the first 6 months and a positive effect in the subsequent 6 months. ${ }^{27}$

When comparing studies by dropout rate, there was no difference in overall QoL between the studies with high $(>50 \%)$ and low $(<50 \%)$ rates (Fig. 3). Results were comparable for each domain (data not presented). 


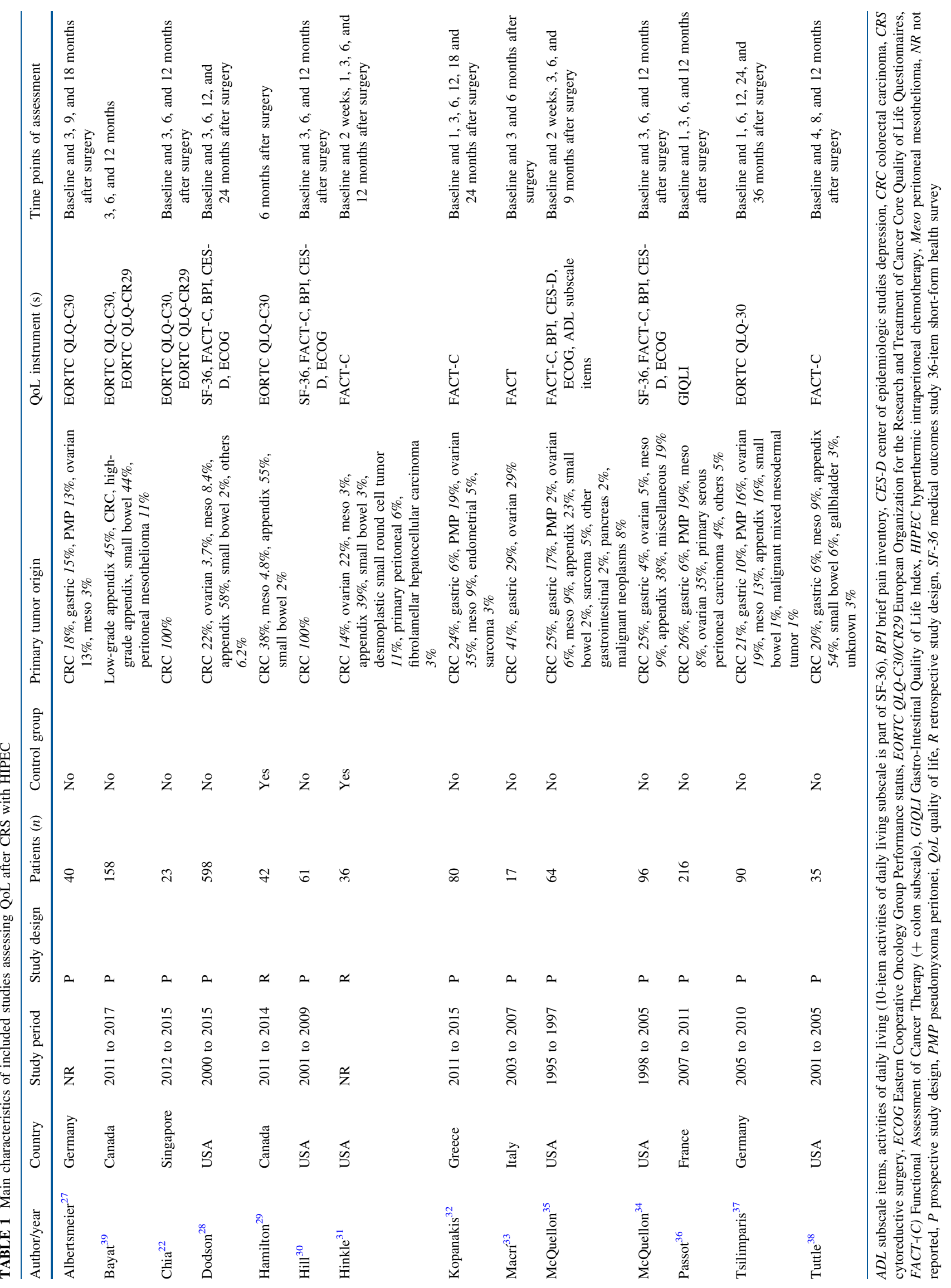




\section{Drop-out 12 months after CRS+ HIPEC}

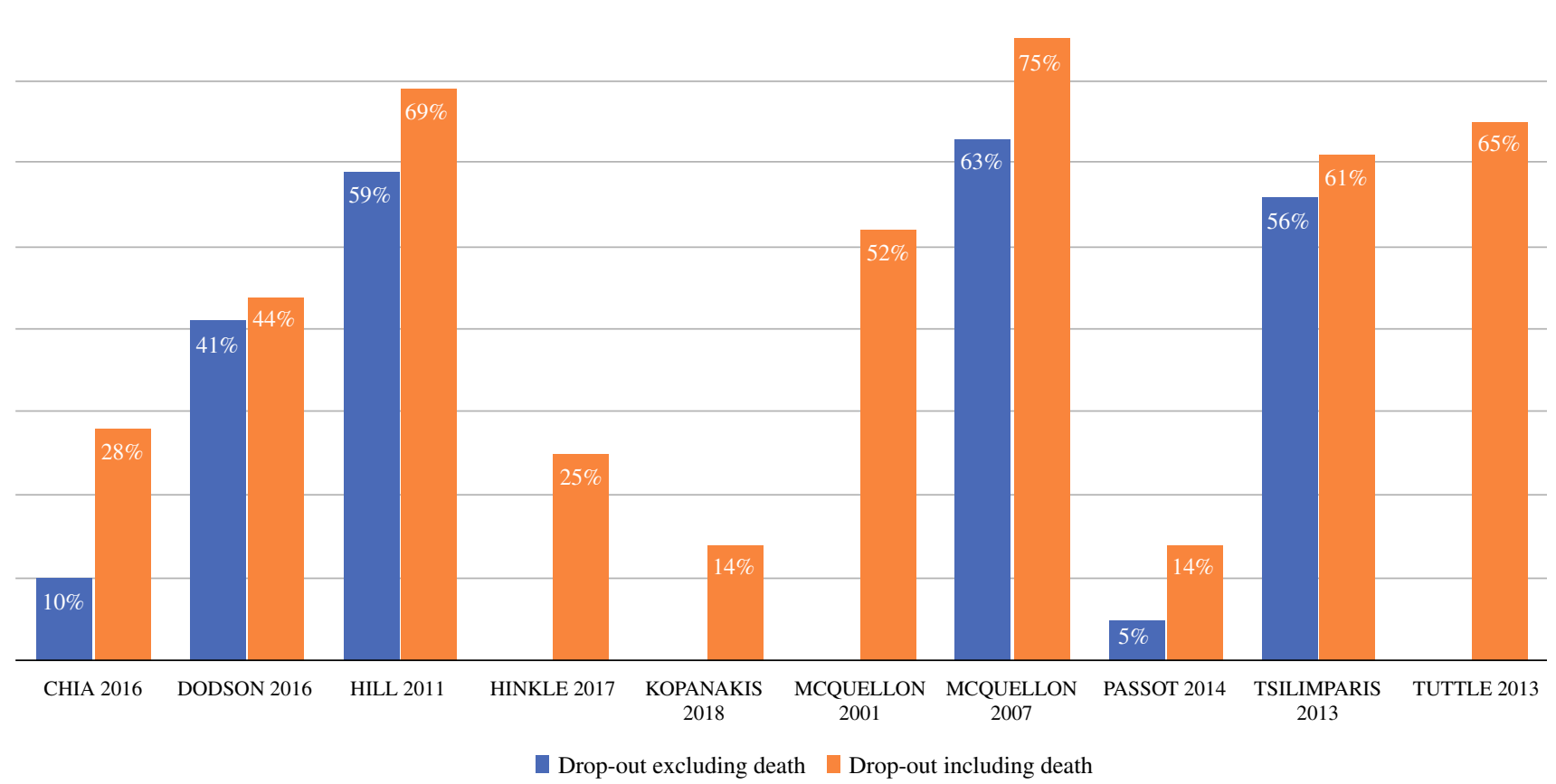

FIG. 2 Dropout rates. $C R S$ cytoreductive surgery, HIPEC hyperthermic intraperitoneal chemotherapy

FIG. 3 Overall QoL. EORTC $Q L Q-C 30$ European

Organisation for Research and Treatment of Cancer Quality of Life of Cancer Patients, FACT Functional Assessment of Cancer Therapy, GIQLI Gastrointestinal Quality of Life index, $Q o L$ quality of life

\section{Overall Health (<50\% Drop-out)}

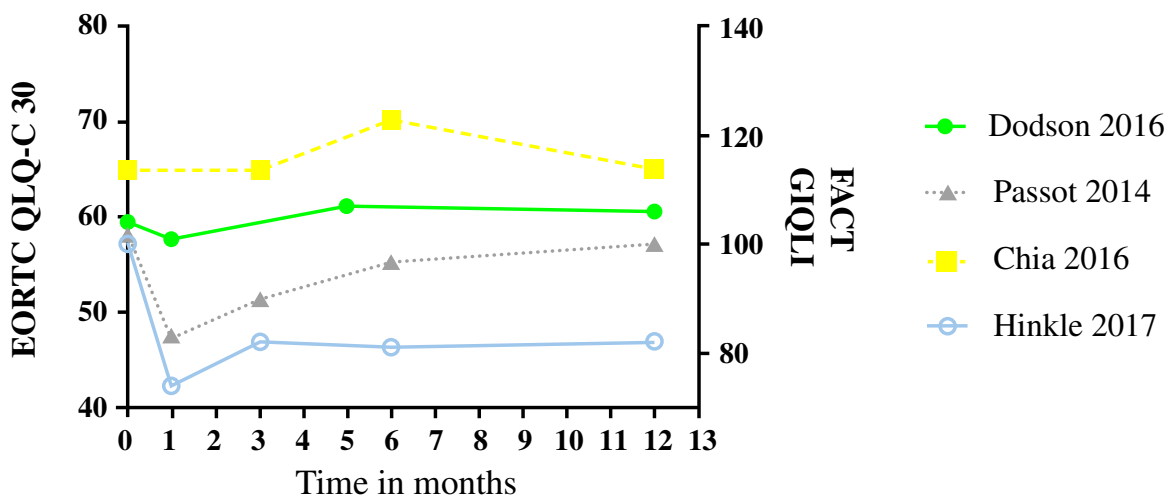

Overall Health (<50\% Drop-out or Drop-out unknown)

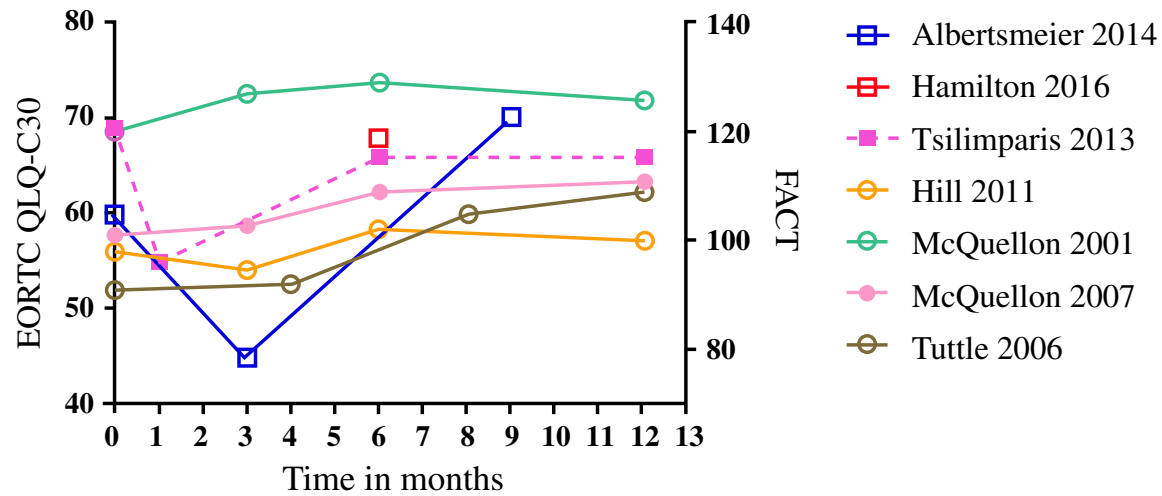



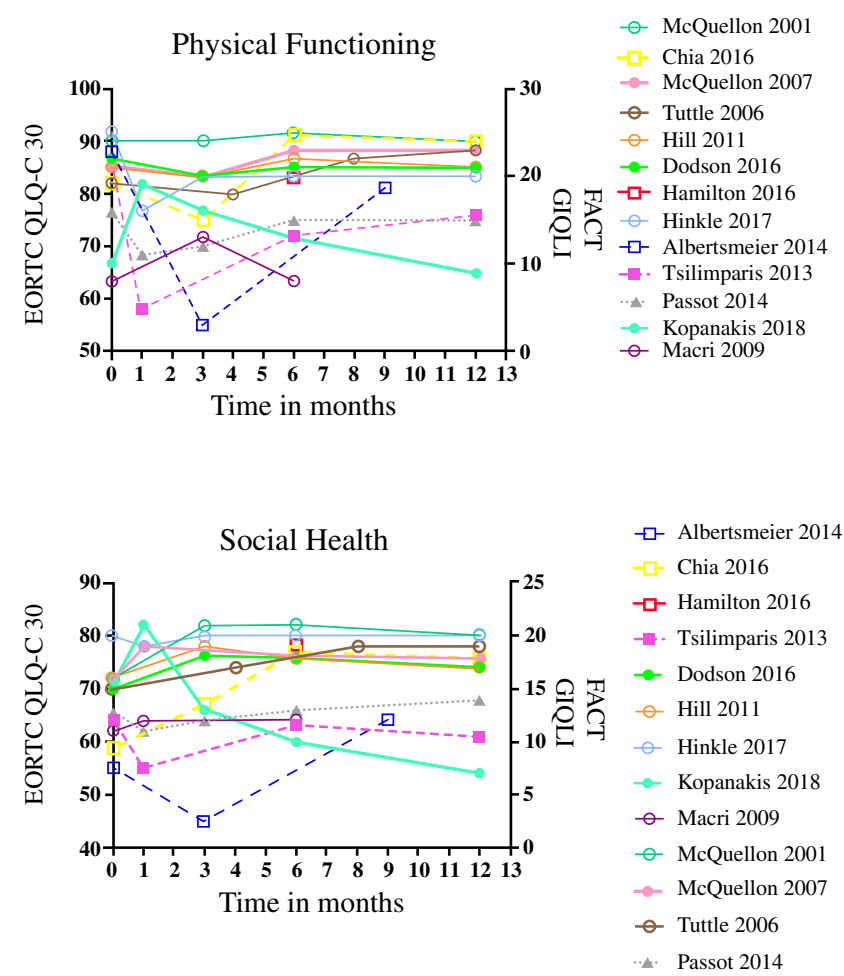

Cognitive functioning

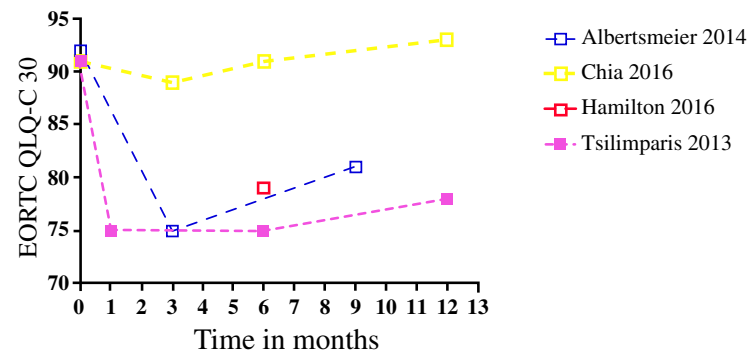

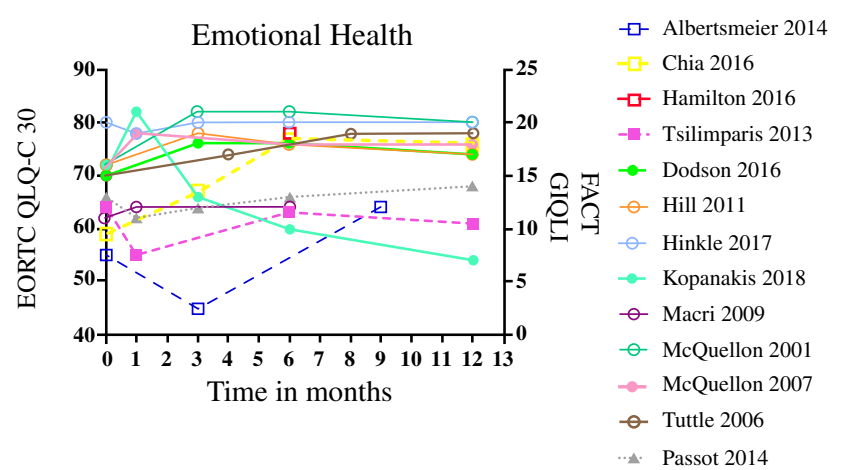

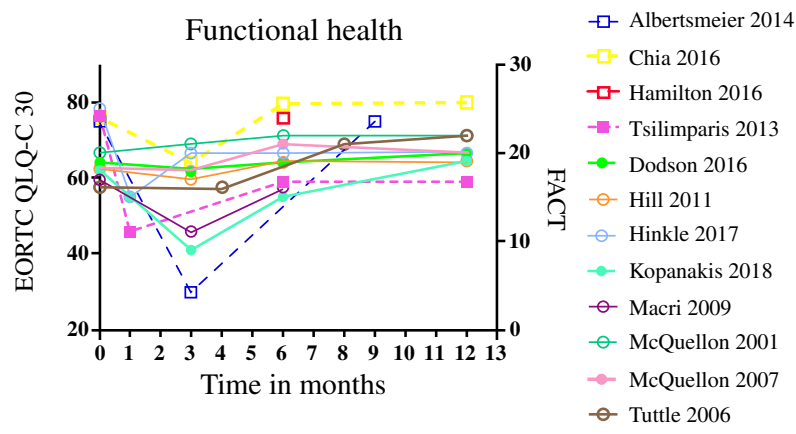

FIG. 4 QoL by domain. EORTC $Q L Q-C 30$ European Organisation for Research and Treatment of Cancer Quality of Life of Cancer Patients, FACT Functional Assessment of Cancer Therapy, GIQLI Gastrointestinal Quality of Life index, QoL quality of life

\section{DISCUSSION}

This systematic review identified several factors that negatively influence QoL after CRS + HIPEC. These factors are higher patient age, female sex, prolonged operation time, extensive disease (higher PCI), more residual disease after surgery (higher CC score), adjuvant chemotherapy, postoperative complications, stoma placement, and disease recurrence. It was striking that dropout rates did not affect these results. Overall, most patients experienced a significant decline in a broad range of QoL domains during the first months after CRS + HIPEC, but generally recovered to preoperative levels by $6-12$ months after surgery.

The recovery process over the first year after CRS + HIPEC appears to be promising, but these results should be interpreted with caution. Although we found no difference in QoL among studies based on their dropout rates, we must remember that our results only apply to patients who remained in the studies. Only 4 of 14 studies reported QoL data based on over half of their enrolled populations, and patient deaths only explained a small amount of the dropouts. Most dropouts were for other reasons, such as patients being too weak to continue with the study due to either disease recurrence or significant symptomatology. ${ }^{30}$ Consequently, QoL may be overestimated after CRS + HIPEC. However, only one study reported on the reasons for dropout, so it is difficult to evaluate the possible overestimation of QoL.

Another relevant issue was that the included studies were of mediocre quality based on the MINORS criteria. This included having small sample sizes (only five studies included more than 80 patients) and heterogeneous patient groups with a variety of primary tumors. The reported QoL 
TABLE 2 Overall trend of impact of CRS with HIPEC on different symptoms short- and long-term

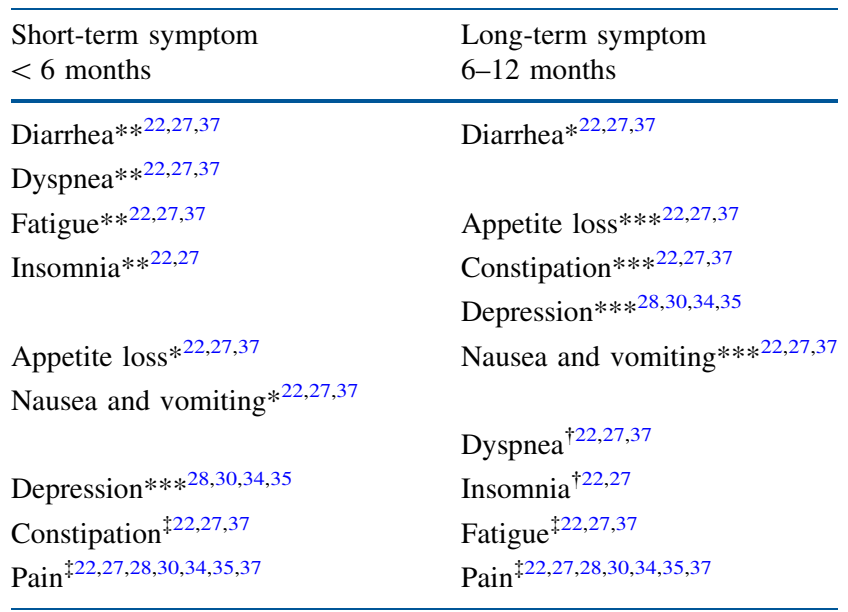

CRS cytoreductive surgery, HIPEC hyperthermic intraperitoneal chemotherapy

*Nonsignificant increase of symptom compared with prior assessment (e.g., preoperative assessment or within 6 months after surgery assessment)

**Significant increase of symptom compared with preoperative assessment $(p<0.005)$

***Nonsignificant decrease of symptom compared with prior assessment

${ }^{\dagger}$ Significant decrease of symptom compared with prior assessment

${ }^{*}$ Increase of symptom above preoperative assessment

of patients might be more determined by the different tumor types and stages than the other factors. To evaluate the impact of tumor type and stage on QoL, studies are needed that stratify their data by tumor type and stage. Moreover, some important factors that could have affected QoL (e.g., patient characteristics, tumor characteristics, HIPEC regimens, and postoperative morbidity) were only analyzed in a few studies. ${ }^{22,31,33}$ It was also notable that there had been no consideration as to whether some factors were associated with each other.

A strength of the included studies was that they all used standardized and validated questionnaires. However, the results may fail to give a comprehensive view of the QoL of patients after CRS + HIPEC specifically, not least because the applied questionnaires were not designed for this purpose. Furthermore, the studies used different validated questionnaires to assess QoL and measured QoL at differing times. In studies using in-depth semi structured interviews to collect QoL data after CRS + HIPEC, physical symptoms were reported to persist in at least half of the patients between 6 and 12 months after surgery.
These included chronic pain, diet restrictions, ongoing gastrointestinal problems, and sleep difficulties. ${ }^{40}$ In other cases, patients described crying spells, depression, and stoma-related problems (e.g., social issues, negative effect on intimate relationships, and constant reminder of disease), ${ }^{41}$ as well as uncertainty about the future or death. ${ }^{42}$ These symptoms and their impact on QoL can be missed when using questionnaires that are not disease specific. This may be rectified by developing a standardized and validated questionnaire for use after CRS + HIPEC.

Most included studies described that QoL domains returned to baseline levels after CRS + HIPEC. However, it must be remembered that baseline QoL levels were measured shortly before the operation, when patients might have already been suffering from clinical symptoms of their disease. Therefore, QoL may have been already lower compared with the QoL before the onset of the disease, as it has been reported in patients suffering from malignant ascites before CRS + HIPEC. ${ }^{35}$ Therefore, it is questionable whether we should consider a return to baseline QoL a sufficient metric. It has been shown that patients who received CRS + HIPEC and remained disease-free during follow-up scored higher on overall health than patients who developed untreatable recurrent or metastatic disease. ${ }^{43}$ After 1 year of follow-up, their QoL scores were also reported as comparable to those of patients with cancer who undergo surgery without HIPEC and to those of patients who are disease free and functioning well. ${ }^{44,45}$ However, QoL scores are still reported to be lower than in the general population. ${ }^{46}$ Clinicians and patients must therefore consider that QoL may remain lower than before the disease first developed. When making clinical decisions, we advocate that practitioners consider the patient's expectations and their perspectives regarding QoL, life goals, and other influential factors.

In conclusion, this review shows that QoL after CRS + HIPEC tends to be negatively affected by certain patient characteristics, procedure-specific outcomes, and postoperative disease. Notably, study dropout rates did not affect these factors. Although most patients experience a significant decline in a broad range of QoL domains during the first few months after CRS + HIPEC, they generally recover to preoperative levels by 6-12 months. Future research should now focus on study designs that can describe the profound experiences of patients who have undergone CRS + HIPEC. Given that factors affecting QoL can only be partially influenced, it is essential that patients receive detailed and honest counseling about these outcomes before CRS + HIPEC. 
TABLE 3 Determinants of QoL after CRS with HIPEC

\begin{tabular}{|c|c|c|}
\hline Determinant & Result & References \\
\hline Age (years) & Younger age leads to a quicker recovery of QoL & Kopanakis $^{32,}$ Macrí ${ }^{33}$ \\
\hline Sex & Female sex is associated with lower QoL and lower emotional health & Albertsmeier $^{27}$, Kopanakis ${ }^{32}$ \\
\hline $\begin{array}{l}\text { Primary tumor } \\
\text { site }\end{array}$ & $\begin{array}{l}\text { Ovarian carcinomas show slower recover of QoL } \\
\text { Colon and gastric cancer show lower QoL but higher emotional health }\end{array}$ & Albertsmeier $^{27}$, Macri $^{33}$ \\
\hline $\begin{array}{l}\text { Malignant } \\
\text { ascites }\end{array}$ & $\begin{array}{l}\text { Patients with malignant ascites show lower baseline scores of QoL but, after } \\
\text { operation, immediate increase of QoL } \\
\text { Patients without malignant ascites experience a decrease of QoL at } 3 \text { months, } \\
\text { after which recovery begins }\end{array}$ & McQuellon $^{35}$ \\
\hline $\begin{array}{l}\text { Prolonged } \\
\text { operation time }\end{array}$ & Longer duration of surgery is associated with diminished QoL & Chia $^{22,}$ Passot $^{36}$ \\
\hline PCI & $\begin{array}{l}\text { Patients with higher PCI suffer from greater decrease in QoL } \\
\text { One study did not find a difference in QoL in patients with high or low PCI }\end{array}$ & Chia $^{22,}$ Kopanakis $^{32,}$ Passot $^{36,}$ Albertsmeier ${ }^{27}$ \\
\hline CC score & Patients with higher CC score experience higher decrease of QoL & Chia $^{22,}$ Kopanakis $^{32,}$ Macri $^{33,}$ Passot $^{36}$ \\
\hline $\begin{array}{l}\text { Type of bowel } \\
\text { resection }\end{array}$ & $\begin{array}{l}\text { Type of bowel resection does not influence QoL } \\
\text { Social function scores are lower in patients with lower anterior resection }\end{array}$ & Bayat $^{39}$ \\
\hline $\begin{array}{l}\text { Adjuvant } \\
\text { chemotherapy }\end{array}$ & $\begin{array}{l}\text { Patients who receive adjuvant chemotherapy show lower QoL after } \\
\text { CRS + HIPEC }\end{array}$ & Macri $^{33,}$ Chia $^{22}$ \\
\hline Complication & Patients who experienced complications show slower recovery of QoL & Tuttle $^{38,}$Hamilton $^{29}$ \\
\hline $\begin{array}{l}\text { Presence of } \\
\text { Stoma }\end{array}$ & Presence of a stoma is negatively associated with QoL & Chia $^{22,}$ Kopanaki $^{32,}$ Passot $^{36}$ \\
\hline Recurrence & Recurrence within 1 year leads to a lower QoL & $\mathrm{Chia}^{22,}$ Passot $^{36}$ \\
\hline
\end{tabular}

CC score Completeness of Cytoreduction Score, CRS cytoreductive surgery, HIPEC hyperthermic intraperitoneal chemotherapy, PCI Peritoneal Cancer Index, $Q o L$ quality of life

ACKNOWLEDGMENTS We thank Dr Robert Sykes (www.doc tored.org.uk) for providing editorial and language services.

DISCLOSURE The authors have no conflicts of interest to declare.

OPEN ACCESS This article is licensed under a Creative Commons Attribution 4.0 International License, which permits use, sharing, adaptation, distribution and reproduction in any medium or format, as long as you give appropriate credit to the original author(s) and the source, provide a link to the Creative Commons licence, and indicate if changes were made. The images or other third party material in this article are included in the article's Creative Commons licence, unless indicated otherwise in a credit line to the material. If material is not included in the article's Creative Commons licence and your intended use is not permitted by statutory regulation or exceeds the permitted use, you will need to obtain permission directly from the copyright holder. To view a copy of this licence, visit http://creativecommons. org/licenses/by/4.0/.

\section{REFERENCES}

1. Al-Shammaa HA, Li Y, Yonemura Y. Current status and future strategies of cytoreductive surgery plus intraperitoneal hyperthermic chemotherapy for peritoneal carcinomatosis. World $J$ Gastroenterol. 2008;14(8):1159-1166.

2. Cao C, Yan TD, Black D, Morris DL. A systematic review and meta-analysis of cytoreductive surgery with perioperative intraperitoneal chemotherapy for peritoneal carcinomatosis of colorectal origin. Ann Surg Oncol. 2009;16(8):2152-2165.
3. Deraco M, Baratti D, Kusamura S. Morbidity and quality of life following cytoreduction and HIPEC. Cancer Treat Res. 2007;134:403-418.

4. Sadeghi B, Arvieux C, Glehen O, et al. Peritoneal carcinomatosis from non-gynecologic malignancies: Results of the EVOCAPE 1 multicentric prospective study. Cancer. 2000;88(2):358-363.

5. Jayne DG, Fook S, Loi C, Seow-Choen F. Peritoneal carcinomatosis from colorectal cancer. $\mathrm{Br} J$ Surg. 2002;89(12):1545-1550.

6. Mahteme H, Hansson J, Berglund A, et al. Improved survival in patients with peritoneal metastases from colorectal cancer: A preliminary study. Br J Cancer. 2004;90(2):403-407.

7. Sugarbaker PH. New standard of care for appendiceal epithelial neoplasms and pseudomyxoma peritonei syndrome? Lancet Oncol. 2006;7(1):69-76.

8. Harmon RL, Sugarbaker PH. Prognostic indicators in peritoneal carcinomatosis from gastrointestinal cancer. Int Semin Surg Oncol. 2005;2(1):3-7800-2-3.

9. Levine EA, Stewart 4th JH, Russell GB, Geisinger KR, Loggie BL, Shen P. Cytoreductive surgery and intraperitoneal hyperthermic chemotherapy for peritoneal surface malignancy: Experience with 501 procedures. $J$ Am Coll Surg. 2007;204(5):943-53; (discussion 953-5).

10. Smeenk RM, Verwaal VJ, Antonini N, Zoetmulder FA. Survival analysis of pseudomyxoma peritonei patients treated by cytoreductive surgery and hyperthermic intraperitoneal chemotherapy. Ann Surg. 2007;245(1):104-109.

11. Sugarbaker PH, Cunliffe WJ, Belliveau J, et al. Rationale for integrating early postoperative intraperitoneal chemotherapy into the surgical treatment of gastrointestinal cancer. Semin Oncol. 1989;16(4 Suppl 6):83-97.

12. Elias D, Gilly F, Boutitie F, et al. Peritoneal colorectal carcinomatosis treated with surgery and perioperative intraperitoneal 
chemotherapy: Retrospective analysis of 523 patients from a multicentric french study. J Clin Oncol. 2010;28(1):63-68.

13. Esquivel J, Elias D, Baratti D, Kusamura S, Deraco M. Consensus statement on the loco regional treatment of colorectal cancer with peritoneal dissemination. J Surg Oncol. 2008;98(4):263-267.

14. Chua TC, Yan TD, Saxena A, Morris DL. Should the treatment of peritoneal carcinomatosis by cytoreductive surgery and hyperthermic intraperitoneal chemotherapy still be regarded as a highly morbid procedure? A systematic review of morbidity and mortality. Ann Surg. 2009;249(6):900-907.

15. Glehen O, Kwiatkowski F, Sugarbaker PH, et al. Cytoreductive surgery combined with perioperative intraperitoneal chemotherapy for the management of peritoneal carcinomatosis from colorectal cancer: A multi-institutional study. J Clin Oncol. 2004;22(16):3284-3292.

16. Gomez Portilla A, Barrios P, Rufian S, et al. Management of peritoneal surface malignancy with cytoreductive surgery and perioperative intraperitoneal chemotherapy. Eur J Surg Oncol. 2006;32(6):628-631.

17. Robella M, Vaira M, Cinquegrana A, De Simone M. Cytoreductive surgery and hyperthermic intraperitoneal chemotherapy: Morbidity and postoperative outcomes. Minerva Chir. 2019;74(3):195-202.

18. van Leeuwen BL, Graf W, Pahlman L, Mahteme H. Swedish experience with peritonectomy and HIPEC. HIPEC in peritoneal carcinomatosis. Ann Surg Oncol. 2008;15(3):745-753.

19. Verwaal VJ, van Ruth S, de Bree E, et al. Randomized trial of cytoreduction and hyperthermic intraperitoneal chemotherapy versus systemic chemotherapy and palliative surgery in patients with peritoneal carcinomatosis of colorectal cancer. J Clin Oncol. 2003;21(20):3737-3743.

20. Seretis C, Youssef H. Quality of life after cytoreductive surgery and intraoperative hyperthermic intraperitoneal chemotherapy for peritoneal surface malignancies: A systematic review. Eur J Surg Oncol. 2014;40(12):1605-1613.

21. Shan LL, Saxena A, Shan BL, Morris DL. Quality of life after cytoreductive surgery and hyperthermic intra-peritoneal chemotherapy for peritoneal carcinomatosis: A systematic review and meta-analysis. Surg Oncol. 2014;23(4):199-210.

22. Chia CS, Tan GHC, Lim C, Soo KC, Teo MCC. Prospective quality of life study for colorectal cancer patients with peritoneal carcinomatosis undergoing cytoreductive surgery and hyperthermic intraperitoneal chemotherapy. Ann Surg Oncol. 2016;23(9):2905-2913.

23. Moher D, Liberati A, Tetzlaff J, Altman DG, PRISMA Group. Preferred reporting items for systematic reviews and meta-analyses: the PRISMA statement. $J$ Clin Epidemiol. 2009;62(10):1006-1012.

24. Stroup DF, Berlin JA, Morton SC, et al. Meta-analysis of observational studies in epidemiology: A proposal for reporting. Metaanalysis of observational studies in epidemiology (MOOSE) group. JAMA. 2000;283(15):2008-2012.

25. Slim K, Nini E, Forestier D, Kwiatkowski F, Panis Y, Chipponi J. Methodological index for non-randomized studies (minors): development and validation of a new instrument. ANZ J Surg. 2003;73(9):712-716.

26. Landis JR, Koch GG. The measurement of observer agreement for categorical data. Biometrics. 1977;33(1):159-174.

27. Albertsmeier M, Hauer A, Niess H, Werner J, Graeb C, Angele MK. Quality of life in peritoneal carcinomatosis: a prospective study in patients undergoing cytoreductive surgery and hyperthermic intraperitoneal chemotherapy (HIPEC). Dig Surg. 2014;31(4-5):334-340.

28. Dodson RM, McQuellon RP, Mogal HD, et al. Quality-of-life evaluation after cytoreductive surgery with hyperthermic intraperitoneal chemotherapy. Ann Surg Oncol. 2016;23:S772S783.

29. Hamilton TD, Taylor EL, Cannell AJ, McCart JA, Govindarajan A. Impact of major complications on patients' quality of life after cytoreductive surgery and hyperthermic intraperitoneal chemotherapy. Ann Surg Oncol. 2016;23(9):2946-2952.

30. Hill AR, McQuellon RP, Russell GB, Shen P, Stewart IV JH, Levine EA. Survival and quality of life following cytoreductive surgery plus hyperthermic intraperitoneal chemotherapy for peritoneal carcinomatosis of colonic origin. Ann Surg Oncol. 2011;18(13):3673-3679.

31. Hinkle NM, Botta V, Sharpe JP, Dickson P, Deneve J, Munene G. The impact of early recurrence on quality of life after cytoreduction with HIPEC. Am Surg. 2017;83(6):633-639.

32. Kopanakis N, Argyriou E-, Vassiliadou D, et al. Quality of life after cytoreductive surgery and HIPEC: A single centre prospective study. J B U ON. 2018;23(2):488-493.

33. Macrí A, Maugeri I, Trimarchi G, et al. Evaluation of quality of life of patients submitted to cytoreductive surgery and hyperthermic intraperitionaeal chemotherapy for peritioneal carcinosis of gastrointestinal and ovarian origin and identification of factors influencing outcome. In Vivo. 2009;23(1):147-150.

34. McQuellon RP, Danhauer SC, Russell GB, et al. Monitoring health outcomes following cytoreductive surgery plus intraperitoneal hyperthermic chemotherapy for peritoneal carcinomatosis. Ann Surg Oncol. 2007;14(3):1105-1113.

35. McQuellon R, Loggie B, Fleming R, Russell G, Lehman A, Rambo T. Quality of life after intraperitoneal hyperthermic chemotherapy (IPHC) for peritoneal carcinomatosis. Eur J Surg Oncol. 2001;27(1):65-73.

36. Passot G, Bakrin N, Roux AS, et al. Quality of life after cytoreductive surgery plus hyperthermic intraperitoneal chemotherapy: A prospective study of 216 patients. Eur J Surg Oncol. 2014;40(5):529-535.

37. Tsilimparis N, Bockelmann C, Raue W, et al. Quality of life in patients after cytoreductive surgery and hyperthermic intraperitoneal chemotherapy: Is it worth the risk? Ann Surg Oncol. 2013;20(1):226-232.

38. Tuttle TM, Zhang Y, Greeno E, Knutsen A. Toxicity and quality of life after cytoreductive surgery plus hyperthermic intraperitoneal chemotherapy. Ann Surg Oncol. 2006;13(12):1627-1632.

39. Bayat Z, Taylor EL, Bischof DA, McCart JA, Govindarajan A. Impairments in bowel function, social function and quality of life after cytoreductive surgery and hyperthermic intraperitoneal chemotherapy. Ann Surg Oncol. 2020;27(1):124-131.

40. Francescutti VA, Maciver AH, Stewart E, et al. Characterizing the patient experience of CS/HIPEC through in-depth interviews with patients: Identification of key concepts in the development of a patient-centered program. Ann Surg Oncol. 2019;26(4):1063-1070.

41. Leo Swenne C, Cederholm K, Gustafsson M, Arakelian E. Postoperative health and patients' experiences of efficiency and quality of care after cytoreductive surgery and hyperthermic intraperitoneal chemotherapy, two to six months after surgery. Eur J Oncol Nurs. 2015;19(2):191-197.

42. Eriksson H, Haglund K, Leo Swenne C, Arakelian E. Patients' experiences of postoperative health related to cytoreductive surgery and hyperthermic intraoperative chemotherapy. J Clin Nurs. 2014;23(1-2):201-210.

43. Chia CS, Tan WJ, Wong JFS, et al. Quality of life in patients with peritoneal surface malignancies after cytoreductive surgery and hyperthermic intraperitoneal chemotherapy. Eur J Surg Oncol. 2014;40(8):909-916.

44. Duzgun O, Sarici IS, Gokcay S. Short-term quality of life after cytoreductive surgery and hyperthermic intraperitoneal chemotherapy. Istanb Med J. 2018;19(2):143-146. 
45. Tan WJ, Wong JFS, Chia CS, Tan GHC, Soo KC, Teo MCC. Quality of life after cytoreductive surgery and hyperthermic intraperitoneal chemotherapy: An asian perspective. Ann Surg Oncol. 2013;20(13):4219-4223.

46. Schmidt U, Dahlke M, Klempnauer J, Schlitt H, Piso P. Perioperative morbidity and quality of life in long-term survivors following cytoreductive surgery and hyperthermic intraperitoneal chemotherapy. EJSO. 2005;31(1):53-58.

Publisher's Note Springer Nature remains neutral with regard to jurisdictional claims in published maps and institutional affiliations. 